\title{
ANÁLISE DA RELAÇÃO ENTRE MODELO DE GESTÃO PORTUÁRIA E EFICIÊNCIA EM PORTOS DE CONTÊINERES
}

\section{ANALYSIS OF THE RELATIONSHIP BETWEEN PORT MANAGEMENT MODEL AND EFFICIENCY IN CONTAINER PORTS}

\author{
Priscila Milani ${ }^{1}$; Guilherme Bergmann Borges Vieira ${ }^{2}$; Arturo Monfort Mulinas ${ }^{3}$; \\ Fábio Verruck ${ }^{4}$; Roberto Birch Gonçalves ${ }^{5}$ \\ ${ }^{1}$ Universidade de Caxias do Sul (UCS) - Caxias do Sul - Brasil \\ prikamil@hotmail.com \\ ${ }^{2}$ Universidade de Caxias do Sul (UCS) - Caxias do Sul - Brasil \\ gbbvieir@ucs.br \\ ${ }^{3}$ Fundación Valenciaport (FV) - Valencia - Espanha \\ amonfort@fundacion.valenciaport.com \\ ${ }^{4}$ Universidade de Caxias do Sul (UCS) - Caxias do Sul - Brasil \\ fverruck@ucs.br \\ ${ }^{5}$ Universidade de Caxias do Sul (UCS) - Caxias do Sul - Brasil \\ rbgoncal@ucs.br
}

\begin{abstract}
Resumo
Atualmente, os portos fazem parte de uma complexa cadeia logística com variadas combinações relacionadas à sua titularidade, modelo de gestão e governança. Considerando esse contexto, esta pesquisa tem por objetivo analisar a relação entre modelo de gestão portuária e eficiência em portos de contêineres. Para tanto, tomando-se como base os dados dos 50 maiores portos de contêineres do mundo, foi aplicado um modelo DEA VRS orientado a outputs para identificar os portos mais eficientes e foram analisados seus respectivos modelos de gestão. A partir desses resultados, os portos foram agrupados segundo o grau de eficiência (alto, médio-alto, médio-baixo ou baixo) e segundo o modelo de gestão (totalmente público, predominantemente público, predominantemente privado ou totalmente privado). Os resultados indicaram que não há relação direta entre modelo de gestão portuária e eficiência, uma vez que entre os seis portos com grau de eficiência alta foram identificados diferentes modelos de gestão.
\end{abstract}

Palavras-chave: Portos de Contêineres; Gestão; Modelo; Eficiência; Análise Envoltória de dados.

\section{Introdução}

De acordo com a Conferência das Nações Unidas para o Comércio e Desenvolvimento (UNCTAD, 2011), de 1980 até 2011, o transporte de contêineres, em toneladas, aumentou sua participação no total de toneladas movimentadas através dos portos em 1.348\%, de 102 milhões de toneladas transportadas em 1980 para 1,48 bilhões em 2011. Além desse recente crescimento, destacam-se as cargas conteinerizadas como um importante componente das operações portuárias, 
pois possuem um alto valor agregado e exigem um serviço rígido, em função das necessidades associadas aos diferentes tipos de carga conteinerizáveis e da crescente complexidade na cadeia logística global.

Devido ao crescimento do setor portuário, os armadores têm procurado aumentar sua eficiência para maximizar sua lucratividade. Para alcançar essa eficiência, os armadores exigem que os portos também aprimorem a qualidade e a eficiência de seus serviços. Com isso, os portos acabam buscando um aperfeiçoamento em sua administração e gestão para assim tornarem-se competitivos (VALENTINE; GRAY, 2002).

Considerando a abordagem microeconômica e os referidos modelos de gestão portuária, percebe-se, com o aumento da competitividade, um aparente entusiasmo a favor da privatização dos portos. Entretanto, existem também algumas opiniões contrárias à relação entre privatização portuária e eficiência, já que não há uma teoria econômica tradicional nem uma prova empírica que possa comprovar a veracidade de que a privatização dos portos levará inevitavelmente a um melhor desempenho (CULLINANE; JI; WANG, 2005).

Apesar do crescimento do setor, muitas vezes os investimentos e a gestão portuária não acompanham seu desenvolvimento, ocasionando o que se pode chamar de ineficiência portuária. A relação entre os modelos portuários e a eficiência vem sendo amplamente discutida na literatura, porém não existem evidências conclusivas de que uma maior participação privada nos portos leve a uma maior eficiência ou de que determinado modelo de gestão favoreça a performance portuária. Dado esse contexto, o objetivo do presente estudo é analisar a relação entre os modelos de gestão portuárias e a eficiência em portos de contêineres. Para tanto, foi selecionada uma amostra com os maiores portos de contêineres do mundo; foram verificados os modelos de gestão adotados por cada um desses portos; foi analisada a eficiência dos portos da amostra, mediante a aplicação da Análise Envoltória de Dados (DEA); e foi discutida a relação entre modelo de gestão e eficiência portuária nesses portos.

Com base nos dados coletados, a pesquisa avaliou se a eficiência dos maiores portos de contêineres mundiais está relacionada com seu modelo de gestão portuária. O objetivo é apontar pontos relevantes que poderão ser discutidos, avaliados e trabalhados pelos gestores portuários, a fim de auxiliá-los na implementação de mudanças e estratégias no âmbito portuário, visando orientar projetos de expansão e definição da capacidade portuária. Este trabalho também revela conclusões úteis para os órgãos intervenientes na área e para pesquisadores interessados no tema.

O presente artigo está organizado em quatro seções. Na primeira seção é apresentado o referencial teórico, dando-se ênfase nos modelos de gestão portuária e na eficiência portuária. $\mathrm{Na}$ segunda seção são apresentados os procedimentos metodológicos utilizados no trabalho. Em seguida, são apresentados os resultados e as considerações finais do trabalho. 


\section{Referencial teórico}

Neste capítulo são descritos os principais modelos de gestão portuária, são discutidos os principais conceitos associados à eficiência portuária e são apresentados os principais inputs e outputs considerados em estudos anteriores sobre eficiência portuária que utilizaram a análise envoltória de dados.

\subsection{Modelos de gestão portuária}

Os portos são complexas e dinâmicas entidades, normalmente distintas entre si, onde várias atividades são realizadas por e para diferentes atores e organizações. Tal multifacetada situação tem levado a uma variedade de abordagens operacionais, organizacionais e estratégicas aplicáveis aos portos (BICHOU; GRAY, 2005). Vieira (2013) acrescenta que as atividades dentro de um porto estão relacionadas com muitas outras atividades as quais excedem os limites territoriais dos mesmos, e vão desde a origem da carga (exportação) passando pelo porto e chegando até o seu destino final (importação).

De acordo com Liu (1992), os portos podem ser classificados em quatro tipos considerando sua titularidade e modelo de gerenciamento: (i) service port, (ii) tool port, (iii) landlord port e (iv) private port.

No modelo service port, a autoridade portuária é responsável pelo fornecimento de todas as facilidades e serviços portuários. No modelo tool port, a autoridade portuária pública fornece a infraestrutura e a superestrutura, enquanto a responsabilidade dos serviços fica por conta dos operadores privados, que adquirem o direito de operar através de licenças. Já no modelo landlord port, o domínio do fornecimento da infraestrutura é de responsabilidade da autoridade portuária, enquanto os investimentos com superestrutura e serviços portuários são de responsabilidade de companhias privadas licenciadas. E, por fim, no modelo private port, o fornecimento de todas as facilidades e serviços portuários é de responsabilidade de setores privados.

De acordo com Bichou e Gray (2005), as dissimilaridades entre as instituições dificultam a compreensão dos sistemas portuários, já que existem inúmeros modelos de autoridades usados nos portos mundiais, mesmo entre aqueles que atuam com papéis e funções similares. Um porto pode ser uma empresa de estiva, um terminal, uma autoridade pública, uma companhia privada ou apenas um grupo de diferentes atores e operadores. Os modelos institucionais portuários são diversos, incluindo os modelos de landlord port, service port, tool port e private port, bem como variações e combinações de alguns deles ou todos eles. A principal diferença entre os modelos refere-se aos aspectos de autoridade e gerenciamento da infraestrutura e superestrutura e também as organizações 
de trabalho e mão de obra. No service model, a Autoridade Portuária possui, mantém e desenvolve a infraestrutura e a superestrutura, opera todos os equipamentos e atua sozinha em todas as outras funções comerciais portuárias. Já nos sistemas landlord e tool, as Autoridades Portuárias possuem e desenvolvem a infraestrutura portuária e geralmente a arrendam ao setor privado. Entretanto, enquanto a superestrutura é de propriedade e operada pelo setor privado no modelo landlord, no sistema tool o porto continua proprietário da superestrutura, porém é possível que ele a arrende com propósitos operacionais às companhias privadas. Essa distinção nem sempre fica óbvia. Alguns portos podem restringir a posse da superestrutura aos equipamentos de movimentação de cargas, enquanto outros podem estender tais facilidades aos armazéns e serviços logísticos. A mesma situação se aplica aos trabalhadores, uma vez que, muitas vezes, no modelo tool, as companhias privadas podem contar com trabalhadores do próprio porto.

Bichou e Gray (2005) também apontam algumas forças e fraquezas dos sistemas. No modelo service port, a maior vantagem é a responsabilidade estar toda centralizada em uma só entidade, proporcionando um sistema mais coerente. Porém, a falta de competição interna pode resultar em uma administração ineficiente com pouca inovação e serviços não voltados para os consumidores e o mercado.

No modelo tool port, a força citada é a não ocorrência de investimentos duplicados, já que as instalações são fornecidas pelo setor público. A maior fraqueza deste modelo são os conflitos que podem ser criados entre os operadores de pequena escala ou entre estes operadores e a administração portuária, já que, muitas vezes, a autoridade portuária acaba não investindo em novas e melhores instalações, interferindo desta maneira no desenvolvimento dos operadores privados responsáveis neste modelo pelas operações portuárias.

Já no modelo landlord, a mesma companhia que tem a posse e mantém os equipamentos também é responsável pela operação dos mesmos, o que facilita um planejamento e adaptações melhores ao mercado. Entretanto, o desenvolvimento de mais de um operador privado pode ocasionar excesso de capacidade.

No último modelo, o private service port, a força apontada é a sua orientação para o mercado. Porém, neste modelo corre-se o risco de um comportamento monopólico, particularmente se não houver uma competição intraportos significativa.

Na Figura 1 apresenta-se um resumo conceitual dos principais modelos portuários e suas respectivas administrações.

Quadro 1- Modelos de gestão portuária

\begin{tabular}{|l|l|l|l|l|}
\hline \multirow{2}{*}{ Modelo } & \multicolumn{2}{|c|}{ Investimentos } & \multicolumn{2}{c|}{ Serviços } \\
\cline { 2 - 5 } & Infraestrutura & Superestrutura & Estiva & Outros \\
\hline Service port & Pública & Pública & Pública & Públicos \\
\hline
\end{tabular}




\begin{tabular}{|l|l|l|l|l|}
\hline Tool port & Pública & Pública & Privada & Público-privados \\
\hline Landlord port & Pública-privada & Privada & Privada & Público-privados \\
\hline Private port & Privada & Privada & Privada & Predominantemente privados \\
\hline
\end{tabular}

Fonte: Adaptado de Vieira (2003); Akabane e Gonçalves (2008) e Fundación Valenciaport (2009).

Outra classificação usual para analisar a titularidade e a administração portuária foi a proposta por Baird $(1995,1997)$ e aplicada por Cullinane, Ji e Wang (2005). Nessa proposta, os portos são classificados em quatro categorias, considerando a predominância de atividades dos órgãos administradores e gestores: i) gestão pública, onde não há a interveniência de órgãos privados; ii) gestão pública e privada, com predominância dos órgãos públicos; iii) gestão privada e pública, com predominância dos órgãos privados; e iv) gestão privada, onde não há a interveniência de órgãos públicos. A matriz das funções portuárias pode ser observada na Figura 2.

O princípio dessa classificação, proposta por Baird $(1995,1997)$ é de que o porto deve executar e fornecer as três funções a seguir, estando em domínio público ou privado: i) função reguladora, que consiste na gestão e regulamentação da autoridade portuária; ii) função de proprietário da terra "landowner", que consiste no controle significativo da área portuária, ou seja, da chamada propriedade portuária. Independentemente do tamanho da área portuária, as obrigações envolvidas devem ser a de gerenciar e desenvolver a propriedade portuária, implementando políticas e estratégias para o desenvolvimento físico do porto em termos de superestrutura e, em alguns casos, de infraestrutura; e iii) função de operador, que consiste unicamente na transferência física das mercadorias e passageiros entre o oceano e a terra.

Quadro 2- Matriz das funções portuárias

\begin{tabular}{|lccc|}
\hline \multicolumn{3}{|c|}{ Modelos portuários } & Funções portuárias \\
\hline & Regulação & Propriedade & Operação \\
PÚBLICO & Pública & Pública & Pública \\
PÚBLICO/ privado & Pública & Pública & Privada \\
PRIVADO/público & Pública & Privada & Privada \\
PRIVADO & Privada & Privada & Privada \\
\hline
\end{tabular}

Fonte: Baird $(1995,1997)$

Uma das vantagens do modelo de Baird $(1995,1997)$ é a sua simplicidade de entendimento. Em contrapartida, o modelo do Banco Mundial (1992), além de ser mais complexo, limita o estudo em questão, já que, em muitos casos, um porto pode se posicionar simultaneamente em dois ou mais modelos.

\subsection{Eficiência Portuária}

A eficiência portuária é um importante fator para estimular a competitividade e impulsionar o desenvolvimento regional. Devido ao crescimento do tráfego internacional portuário e as 
mudanças tecnológicas da indústria do transporte marítimo, por exemplo, a conteinerização, serviços interligados da cadeia logística, entre outros, os portos são pressionados a aprimorar e fornecer tecnologia de ponta. Eles também são forçados a melhorar a eficiência portuária e assim fornecer vantagens que irão atrair maior tráfego. Alguns dos maiores desafios dos portos são assegurar o controle do fluxo de trânsito e prevenir o desvio para os portos próximos, incluindo o serviço de movimentação de contêineres e mercadorias mais rapidamente, fornecendo mais equipamentos adequados, reduzindo o tempo de espera nos portos e atrasos, permitindo maior capacidade de armazenamento e garantindo a conexão multimodal com a área terrestre próxima aos portos. Os benefícios da eficiência portuária se estendem além do volume movimentado, pois os mesmos possuem efeitos diretos e indiretos em outras atividades como: seguro marítimo, finanças e logística, devido a sua posição estratégica dentro da cadeia de transportes. Os portos criam valor agregado e empregos, os quais afetam a perspectiva regional e o crescimento urbano (MERK; DANG, 2012).

A eficiência portuária é frequentemente associada com produtividade e desempenho. Entretanto, o foco de estudo se torna restrito, já que a tecnologia operacional e o volume total de cargas movimentadas nos portos não são os únicos indicadores de eficiência. Há fatores adicionais que são associados com a parte organizacional de produção, tal como a maneira como os portos utilizam os inputs, conhecidos como entradas ou nível de recursos, para produzir os níveis de outputs, conhecidos como saídas ou bens produzidos, e se a tecnologia adotada pelos portos são as mais eficientes, sendo cruciais para determinar a eficiência portuária (MERK; DANG, 2012).

As variáveis de inputs e outputs devem refletir os objetivos e processos da produção dos portos de contêineres com a maior precisão possível. A atuação do porto deve estar relacionada com seus objetivos. Por exemplo, será mais provável que um porto utilize equipamentos caros e modernos para aumentar sua produtividade se seu objetivo for aumentar seu rendimento anual de movimentação de carga. Por outro lado, um porto pode optar por utilizar equipamentos mais baratos, se seu objetivo for de aumentar seu lucro (CULLINANE; JI; WANG, 2005).

Os inputs e outputs são as principais variáveis para calcular o grau de eficiência portuária. Na Figura 3 encontram-se 21 estudos realizados entre 2004 a 2013 por pesquisadores da área. Nessa figura estão relacionados os inputs e outputs que foram utilizados nesses estudos. Através da figura, pode-se observar que em todos os estudos foram considerados o rendimento anual em TEUs como o principal ou, na maioria das vezes, como o único output estudado. Por outro lado, os principais inputs considerados foram a área total do terminal $\mathrm{em}^{2}$, o comprimento do cais em metros e o número de equipamentos.

Quadro 3 - Principais inputs e outputs identificados em estudos anteriores

\begin{tabular}{c|l|l|l} 
Autor & Inputs & Outputs & Amostras \\
\hline
\end{tabular}




\begin{tabular}{|c|c|c|c|}
\hline $\begin{array}{l}\text { Barros e Athanassiou } \\
(2004)\end{array}$ & $\begin{array}{l}\text { Mão de obra; } \\
\text { Capital. }\end{array}$ & $\begin{array}{l}\text { Rendimento anual em } \\
\text { TEUs; } \\
\text { Rendimento de navios } \\
\text { por ano; } \\
\text { Movimentação de } \\
\text { fretes; } \\
\text { Movimentação de } \\
\text { cargas. } \\
\end{array}$ & $\begin{array}{l}06 \text { portos } \\
\text { internacionais na } \\
\text { Grécia e Portugal }\end{array}$ \\
\hline Barros (2005) & $\begin{array}{l}\text { Custo da mão de obra; } \\
\text { Capital. }\end{array}$ & $\begin{array}{l}\text { Rendimento anual em } \\
\text { TEUs; } \\
\text { Número de navios. } \\
\end{array}$ & \begin{tabular}{|l|}
10 autoridades \\
portuárias em \\
Portugal
\end{tabular} \\
\hline $\begin{array}{l}\text { Cullinane; Ji; Wang } \\
\text { (2005) }\end{array}$ & $\begin{array}{l}\text { Comprimento do terminal em m; } \\
\text { Área do terminal em hectares; } \\
\text { Número de guindastes do cais; } \\
\text { Número de guindastes do pátio; } \\
\text { Número de equipamentos de } \\
\text { movimentação de contêineres. }\end{array}$ & $\begin{array}{l}\text { Rendimento anual em } \\
\text { TEUs }\end{array}$ & $\begin{array}{l}30 \text { maiores portos em } \\
2001\end{array}$ \\
\hline Lin e Tseng (2005) & $\begin{array}{l}\text { Comprimento do cais em metros; } \\
\text { Número de guindastes; Equipamentos } \\
\text { de carregar e descarregar a carga. }\end{array}$ & $\begin{array}{l}\text { Rendimento anual em } \\
\text { TEUs }\end{array}$ & $\begin{array}{l}27 \text { portos de } \\
\text { contêineres } \\
\text { internacionais }\end{array}$ \\
\hline $\begin{array}{l}\text { Tongzon e Heng } \\
(2005)\end{array}$ & $\begin{array}{l}\text { Comprimento do cais em metros; } \\
\text { Superfície do terminal; } \\
\text { Número de guindastes. }\end{array}$ & $\begin{array}{l}\text { Rendimento anual em } \\
\text { TEUs }\end{array}$ & $\begin{array}{l}25 \text { portos/ terminais } \\
\text { internacionais }\end{array}$ \\
\hline $\begin{array}{l}\text { Cullinane e Song } \\
(2006)\end{array}$ & $\begin{array}{l}\text { Área do terminal em hectares; } \\
\text { Comprimento do cais em metros; } \\
\text { Número de equipamentos. }\end{array}$ & $\begin{array}{l}\text { Rendimento anual em } \\
\text { TEUs }\end{array}$ & Portos europeus \\
\hline $\begin{array}{l}\text { Sun, Yan e Liu } \\
(2006)\end{array}$ & $\begin{array}{l}\text { Área total do terminal em } \mathrm{m}^{2} ; \\
\text { Comprimento do cais em metros; } \\
\text { Número de berços de atracação; } \\
\text { Capacidade de movimentação entre o } \\
\text { navio e o cais do porto; Capacidade de } \\
\text { movimentação entre o cais e o pátio; } \\
\text { Capacidade de armazenagem do porto } \\
\text { e pontos da guarda marinha. }\end{array}$ & $\begin{array}{l}\text { Rendimento anual em } \\
\text { TEUs }\end{array}$ & $\begin{array}{l}83 \text { operadores de } \\
\text { terminais de } \\
\text { contêineres }\end{array}$ \\
\hline $\begin{array}{l}\text { Wang e Cullinane } \\
(2006)\end{array}$ & $\begin{array}{l}\text { Área total do terminal em } \mathrm{m}^{2} ; \\
\text { Comprimento do cais em metros; } \\
\text { Custos com equipamentos. }\end{array}$ & $\begin{array}{l}\text { Rendimento anual em } \\
\text { TEUs }\end{array}$ & $\begin{array}{l}104 \text { terminais } \\
\text { europeus }\end{array}$ \\
\hline $\begin{array}{l}\text { Trujillo e Tovar } \\
(2007)\end{array}$ & $\begin{array}{l}\text { Área de superfície; } \\
\text { Número de empregados. }\end{array}$ & $\begin{array}{l}\text { Rendimento anual em } \\
\text { TEUs; Rendimento do } \\
\text { restante do tráfego dos } \\
\text { outros tipos de carga e } \\
\text { passageiros. }\end{array}$ & $\begin{array}{l}22 \text { autoridades } \\
\text { portuárias europeias }\end{array}$ \\
\hline $\begin{array}{l}\text { Gonzalez e Trujillo } \\
(2008)\end{array}$ & $\begin{array}{l}\text { Números de berços; } \\
\text { Superfície e mão de obra. }\end{array}$ & $\begin{array}{l}\text { Rendimento anual em } \\
\text { TEUs, granéis líquido, } \\
\text { outras cargas e } \\
\text { passageiros. }\end{array}$ & $\begin{array}{l}05 \text { autoridades } \\
\text { portuárias incluindo } \\
17 \text { portos }\end{array}$ \\
\hline Sharma e Yu (2009) & $\begin{array}{l}\text { Área total do terminal em } \mathrm{m}^{2} ; \\
\text { Comprimento do cais em metros; } \\
\text { Número de guindastes do cais do } \\
\text { porto (quay gantry cranes); Número } \\
\text { de guindastes do pátio (yard cranes); } \\
\text { Número de equipamentos de } \\
\text { movimentação de contêineres } \\
\text { (straddle carriers); Número de } \\
\text { empilhadeiras de contêineres } \\
\text { (stacker). }\end{array}$ & $\begin{array}{l}\text { Rendimento anual em } \\
\text { TEUs }\end{array}$ & $\begin{array}{l}70 \text { portos de } \\
\text { contêineres }\end{array}$ \\
\hline
\end{tabular}




\begin{tabular}{|c|c|c|c|}
\hline $\begin{array}{l}\text { Hung, Lu e Wang } \\
(2010)\end{array}$ & $\begin{array}{l}\text { Número de berços; Área total do } \\
\text { terminal em } \mathrm{m}^{2} ; \text { Número de } \\
\text { guindastes do cais (ship-shore } \\
\text { container gantry); Comprimento do } \\
\text { terminal em metros. }\end{array}$ & $\begin{array}{l}\text { Rendimento anual em } \\
\text { TEUs }\end{array}$ & 31 portos da Ásia \\
\hline Sharma e Yu (2010) & $\begin{array}{l}\text { Área total do terminal em } \mathrm{m}^{2} ; \\
\text { Comprimento do cais em metros; } \\
\text { Número de guindastes (cranes); } \\
\text { Número de guindastes de transferência } \\
\text { (transfer cranes); Número de } \\
\text { empilhadeiras de alcance (reach } \\
\text { stacker); Número de equipamentos de } \\
\text { movimentação de contêineres } \\
\text { (straddle carriers). }\end{array}$ & $\begin{array}{l}\text { Rendimento anual em } \\
\text { TEUs }\end{array}$ & $\begin{array}{l}70 \text { portos de } \\
\text { contêineres }\end{array}$ \\
\hline Wu e Goh (2010) & $\begin{array}{l}\text { Área total do terminal em } \mathrm{m}^{2} ; \\
\text { Comprimento do cais em metros; } \\
\text { Número de equipamentos }\end{array}$ & $\begin{array}{l}\text { Rendimento anual em } \\
\text { TEUs }\end{array}$ & $\begin{array}{l}\text { Maiores portos em } \\
2005, \text { incluindo } 21 \\
\text { países de todos os } \\
\text { continentes } \\
\end{array}$ \\
\hline $\begin{array}{l}\text { Lozano, Villa e } \\
\text { Canca (2011) }\end{array}$ & $\begin{array}{l}\text { Área total do terminal em m²; } \\
\text { Comprimento do cais em metros; } \\
\text { Número de guindastes (cranes); } \\
\text { Número de rebocadores (tugs). }\end{array}$ & $\begin{array}{l}\text { Rendimento anual em } \\
\text { TEUs; } \\
\text { Tráfico de cargas em } \\
\text { toneladas/ ano; } \\
\text { Número de navios por } \\
\text { ano. }\end{array}$ & Portos espanhóis \\
\hline Barros (2012) & $\begin{array}{l}\text { Área total do terminal em } \mathrm{m}^{2} ; \\
\text { Comprimento do cais em metros; } \\
\text { Número de trabalhadores. }\end{array}$ & $\begin{array}{l}\text { Rendimento anual em } \\
\text { TEUs; } \\
\text { Graneis sólidos em } \\
\text { toneladas; } \\
\text { Graneis líquidos em } \\
\text { toneladas; } \\
\text { Número de dias para a } \\
\text { movimentação da carga } \\
\text { (atrasos). }\end{array}$ & Portos africanos \\
\hline $\begin{array}{l}\text { Niavis e Tsekeris } \\
(2012)\end{array}$ & $\begin{array}{l}\text { Comprimento do cais em metros; } \\
\text { Número de berços; } \\
\text { Número de guindastes (cranes). }\end{array}$ & $\begin{array}{l}\text { Rendimento anual em } \\
\text { TEUs }\end{array}$ & $\begin{array}{l}30 \text { portos no sudeste } \\
\text { da Europa }\end{array}$ \\
\hline Bichou (2013) & $\begin{array}{l}\text { Área total do terminal em m²; } \\
\text { Comprimento do cais em metros; } \\
\text { Número de portões (gates); } \\
\text { Número de guindastes de cais e pátio, } \\
\text { equipamentos de movimentação de } \\
\text { carga, esteiras caminhões, tratores e } \\
\text { outros veículos internos. } \\
\end{array}$ & $\begin{array}{l}\text { Rendimento anual em } \\
\text { TEUs }\end{array}$ & $\begin{array}{l}420 \text { terminais de } \\
\text { contêineres }\end{array}$ \\
\hline $\begin{array}{l}\text { Trujillo, González e } \\
\text { Jiménez (2013) }\end{array}$ & $\begin{array}{l}\text { Área total do terminal em m²; } \\
\text { Berços em metros; Número de } \\
\text { guindastes }\end{array}$ & $\begin{array}{l}\text { Rendimento anual em } \\
\text { TEUs }\end{array}$ & Portos africanos \\
\hline $\begin{array}{l}\text { Yuen, Zhang e } \\
\text { Cheung (2013) }\end{array}$ & $\begin{array}{l}\text { Área total do terminal em m²; } \\
\text { Comprimento do cais em metros; } \\
\text { Número de berços; Número de } \\
\text { guindastes de cais (quay cranes); } \\
\text { Número de guindastes do pátio (yard } \\
\text { gantries). }\end{array}$ & $\begin{array}{l}\text { Rendimento anual em } \\
\text { TEUs }\end{array}$ & Portos chineses \\
\hline Wanke (2013) & $\begin{array}{l}\text { Número de berços; } \\
\text { Área do armazém em m²; } \\
\text { Área do páito em m². }\end{array}$ & $\begin{array}{l}\text { Rendimento anual em } \\
\text { TEUs; Rendimento } \\
\text { anual do solid bulk em } \\
\text { toneladas. }\end{array}$ & 27 portos brasileiros \\
\hline
\end{tabular}


De acordo com Bichou (2013), existem muitos estudos sobre a relação entre a eficiência e as condições operacionais e organizacionais que apontam discrepância e divergência. Essas divergências são particularmente encontradas nos estudos voltados à análise dos modelos de gestão portuária como determinantes da eficiência portuária, da relação entre a escala econômica e eficiência portuária e do impacto das reformas portuárias na eficiência.

Cullinane, Song e Gray (2002) utilizaram uma amostra de 15 portos de contêineres na Ásia para identificar a relação entre o modelo de gestão e a eficiência. Após a análise dos dados, os autores chegaram à conclusão de que existe uma relação direta entre a participação do setor privado nos portos e o grau de eficiência portuária. Nessa mesma linha, Tongzon e Heng (2005) também concluíram que há relação entre o modelo de gestão e a eficiência portuária. Entretanto, Liu (1995) e Notteboom, Coeck e Van-Den Broech (2000), que estudaram, respectivamente, amostras de 28 portos do Reino Unido e 36 terminais portuários europeus mais quatro terminais de contêineres do Extremo Oriente, chegaram a uma conclusão contrária, de que o modelo de gestão não possui um efeito significativo na eficiência portuária.

Um estudo recente, realizado por Yuen, Zhang e Cheung (2013) em 21 terminais de contêineres chineses, o qual estudou o crescimento desses terminais durante o período de 2003 a 2007, mostrou que os portos em que existe a participação do setor privado apresentam maior eficiência do que os controlados pelo governo.

No entanto, analisando as pesquisas sobre o tema, verifica-se que ainda não há uma certeza da relação entre a eficiência e o modelo de gestão portuária, já que os estudos apresentam opiniões contrárias uns dos outros. Com isso, percebe-se a necessidade de aprofundar as pesquisas nessa área, a fim de buscar alternativas para o aumento da eficiência portuária e o aprimoramento da gestão portuária.

\section{Procedimentos Metodológicos}

Este capítulo tem como objetivo descrever o método utilizado na realização da pesquisa, bem como as técnicas de coleta e análise de dados empregados.

\subsection{Caracterização do Estudo}

O presente estudo pode ser caracterizado como uma pesquisa descritiva, uma vez que caracteriza os modelos de gestão portuária existentes, analisa a eficiência portuária em uma amostra dos cinquenta maiores portos do mundo e descreve a relação entre essas variáveis na amostra estudada. $\mathrm{O}$ método adotado foi o quantitativo, por englobar em seu processo a coleta e análise de dados numéricos e estar centralizado na mensuração de fenômenos. 
Collis e Hussey (2005) destacam dois tipos de estudos: os de corte transversal e os longitudinais. Os estudos de corte transversal são realizados com o intuito de conseguir informações acerca de variáveis em diferentes contextos, porém simultaneamente. A coleta dos dados nesses estudos é realizada apenas uma vez e em um curto espaço de tempo, previamente a sua análise. Já os estudos longitudinais são contínuos e realizados inúmeras vezes. Esse tipo de estudo tem por finalidade a pesquisa da dinâmica do problema, pesquisando, assim, a mesma situação ou pessoas inúmeras vezes. O presente estudo caraceteriza-se como transversal, já que a coleta dos dados foi realizada apenas uma vez e em um curto espaço de tempo, previamente à análise.

\subsection{Definição da Amostra}

A amostra utilizada contemplou os 50 portos de contêineres do mundo com maior movimentação no ano de 2010, segundo o Containerisation International Yearbook (CI) de 2012. Para cada um desses portos, foram coletados alguns inputs e outputs, os quais foram analisados através do método DEA. A amostra foi escolhida por julgamento e se justifica por contemplar diferentes portos, com modelos portuários, inputs e outputs variados. Como o porto de Santos ocupava o quadragésimo terceiro lugar no referido ranking, o mesmo foi incluído no estudo.

O ranking dos maiores portos de contêineres do mundo de 2010, bem como os inputs e outputs, foram retirados do CI com o intuito de padronizar a amostra. O porto de Shenzhen, localizado na China, e quarto colocado no ranking mundial de 2010, foi excluído da pesquisa, pois não foi possível obter todos os dados necessários para a análise.

Wang, Song e Cullinane (2002) argumentam que a maneira mais adequada para analisar a eficiência em portos de contêineres seria separar a amostra por terminais e não considerar todo o porto na análise. Entretanto, os dados disponíveis para as pesquisas normalmente agregam todos os inputs e outputs dos diferentes terminais e consideram o porto como um todo. Por esse motivo, nesta pesquisa foram utilizadas amostras dos portos, sem separá-los por terminais.

\subsection{Procedimentos de Coleta de Dados}

Lakatos e Marconi (2007) dividem as técnicas de coleta de dados em dois grupos: documentação indireta e documentação direta. A documentação indireta abrange a pesquisa documental e bibliográfica. Já a documentação direta abrange a técnica de observação, que possui a finalidade de averiguar fatos ou fenômenos; e a técnica de entrevista, a qual proporciona um diálogo entre o entrevistador e o entrevistado, de modo que o primeiro consiga as informações necessárias para a sua pesquisa. 
Collis e Hussey (2005) denominam as técnicas de fontes de dados. Eles afirmam que há duas fontes de dados principais: a fonte de dados primários e a fonte de dados secundários. As fontes de dados primários coletam os dados diretamente na sua origem, por exemplo, coleta de dados por meio de entrevistas ou observações. Já as fontes de dados secundários buscam dados já existentes, como documentos, livros e relatórios.

Neste estudo foram utilizadas fontes de dados secundários (documentação indireta), uma vez que os inputs e outputs foram retirados do livro Containerisation International Yearbook 2012 (CI). Esses dados são considerados os mais confiáveis e abrangentes disponíveis para esse tipo de pesquisa (CULLINANE; WANG, 2007). Já os dados sobre o modelo de gestão portuária dos portos estudados foram coletados nos seus respectivos sites, bem como em artigos científicos, relatórios e documentos oficiais, o que evidencia o uso de documentaçao indireta. No entanto, para a identificação do modelo e gestão portuária foram utilizadas também fontes de dados primários em casos de coleta de informações complementares, visando confirmar o modelo existente em cada porto.

A classificação dos modelos de gestão portuária escolhida foi a proposta por Baird (1995, 1997) devido a sua simplicidade de entendimento. A classificação proposta pelo Banco Mundial (1992) não foi considerada porque, além de ser mais complexa, limita o estudo em questão, já que, em muitos casos, um porto pode ser classificado simultaneamente em dois ou mais modelos portuários, o que poderia interferir na análise dos dados.

Para atingir o objetivo da pesquisa foram coletados, para cada porto, os seguintes dados: i) o seu modelo de gestão portuária; ii) duas variáveis de inputs; e iii) uma variável de output. Para identificar os inputs e outputs mais utilizados pelos autores da área foram levantados os dados de 21 estudos, realizados por diferentes autores (Figura 3). Dentre os inputs identificados, os mais utilizados foram a área do terminal em $\mathrm{m}^{2} \mathrm{e}$ o comprimento do cais, encontrados em 13 e 11 estudos, respectivamente. Quanto aos outputs, foi considerada a movimentação anual de TEUs, utilizada em todos os 21 estudos. Todos os outros outputs apareceram em, no máximo, três estudos e, por isso, não foram utilizados nesta pesquisa.

Para o cálculo total do comprimento do cais foram somados os cais multipropósito e os de contêineres. Não foram incluídos os cais de carga geral, de barcaças, barcaças de contêineres, cais de balsas e cais roll on/ roll off (ro-ro). A fim de calcular o total da área do terminal $\mathrm{em} \mathrm{m}^{2}$, foram somadas todas as áreas de cada terminal, excluindo apenas os terminais específicos de carga geral, de barcaças, barcaças de contêineres, cais de balsas e cais roll on/roll off (ro-ro).

Os dados coletados foram registrados em uma tabela (Apêndice A). Dessa maneira, as informações estão padronizadas e podem ser localizadas facilmente. 


\subsection{Procedimentos de Análise de Dados}

Conforme identificado em seu delineamento, esta pesquisa é de caráter quantitativo. Para analisar a relação entre o modelo portuário e a eficiência, foi utilizada a Análise Envoltória de Dados ou Data Envelopment Analysis (DEA).

Para Liu (2010), o DEA é um programa matemático de aproximação para estimar a eficiência produtiva. Essa aproximação mapeia a fronteira da produção baseada nas informações dos inputs e outputs. O degrau de ineficiência/ eficiência é avaliado pela distância entre a observação e a fronteira. A vantagem do DEA é de que não há uma teoria estrutural pressuposta aplicada no processo de produção.

Para Charnes, Cooper e Rhodes (1978), o propósito do método DEA é o de medir a eficiência de unidades produtivas, chamadas de Decision Making Units (DMUs), utilizando uma programação matemática na qual a medida da eficiência é resultante da razão da soma ponderada dos bens produzidos (outputs) pelos dados de entrada ou recursos (inputs).

Esse método empírico avalia a eficiência técnica relativa de unidades produtivas, as DMUs, envolvidas em um processo de produção homogêneo como empresas ou portos. Nesse sentido, um porto eficiente é definido como aquele que possui um nível elevado de bens produzidos (outputs) utilizando o mesmo nível de recursos (inputs) comparado com os outros portos estudados, ou aquele que possui níveis baixos de recursos para um dado nível de bens produzidos (outputs). O primeiro tipo de análise é chamado de orientação a outputs, enquanto o segundo é denominado orientação a inputs. A fronteira de produção eficiente é delineada por um conjunto de DMUs eficientes que apontam os portos com os melhores desempenhos. Os ganhos potenciais para um porto com menor eficiência, por exemplo, portos localizados abaixo da fronteira de produção eficiente, são medidos pela sua distância, tanto do ponto de uma abordagem orientada de output como de uma abordagem de input correspondente à fronteira da eficiência (MERK; DANG, 2012).

De acordo com Koster, Balk e Nus (2009), o pressuposto básico do DEA é de que, durante o período de pesquisa, todas as unidades produtivas, DMUs, têm acesso à mesma tecnologia. Tal tecnologia é definida como sendo um conjunto de todas as possíveis combinações de inputs e outputs.

Cullinane e Wang (2007) destacam que uma definição precisa das variáveis de inputs e outputs é extremamente importante para o sucesso e significância da aplicação do DEA. Uma seleção de variáveis erradas ou mal definidas para a coleta e subsequente análise inevitavelmente acabam levando a uma derivação dos resultados que podem facilmente ser mal interpretados e possivelmente ilusórios. 
Sousa Júnior (2010) apresenta os dois principais modelos para a análise do DEA: o modelo CCR, o qual é denominado com as siglas dos nomes de seus criadores, Charnes, Cooper e Rhodes, em 1978, e o modelo BCC, também denominado com as siglas dos seus criadores, Banker, Charnes e Cooper, em 1984. Os modelos CCR e BCC também são conhecidos como CRS, Constant Returns to Scale - escala de retorno constante e VRS, Variable Returns to Scale - Escala de retorno variável, respectivamente. No primeiro modelo, o CCR (CRS), a alteração dos recursos (inputs) acarreta uma alteração proporcional nos bens produzidos (outputs). Esse modelo atua com retorno contínuo à escala de produção. Já no segundo modelo, o BCC (VRS), não é considerada a equivalência entre os recursos (inputs) e os bens produzidos (outputs). Portanto, o BCC (VRS) considera retornos variáveis, desconsiderando a proporcionalidade entre os recursos (inputs) e os bens produzidos (outputs). Em ambos os modelos, a análise pode ser orientada a input ou a output, conforme comentado anteriormente.

Pesquisando os principais estudos que utilizam DEA para a análise da eficiência portuária, observa-se que não existe um consenso entre os autores sobre qual o melhor modelo a ser aplicado. Normalmente, os autores utilizam em suas pesquisas tanto o modelo DEA CRS quanto o modelo VRS. Há também muitos estudos com a utilização de ambos os modelos.

No presente estudo, foi aplicado o modelo DEA VRS orientado a output. A orientação a output justifica-se por ter sido considerada apenas uma variável de saída, a movimentação de TEUs por ano, o que simplifica os procedimentos de análise. A utilização do VRS, por sua vez, justificase por permitir retorno variável de escala, configurando-se como um modelo mais robusto (menos simplista) que o CRS. A aplicação do método DEA foi realizada por meio do Data Envelopment Analysis (computer) Program (DEAP), programa de análise envoltória de dados desenvolvido por Tim Coelli (1996). A versão utilizada nesta pesquisa foi a versão 2.1.

\section{Resultados}

Este capítulo tem como objetivo descrever os resultados da pesquisa. Para tanto, primeiramente é apresentada a classificação dos portos segundo seu grau de eficiência, bem como os modelos de gestão adotados. Posteriormente, são apontados os resultados da eficiência portuária. E, por fim, apresenta-se a síntese dos portos referências (peers).

\subsection{Eficiência (Efficiency Summary)}

Para facilitar a análise dos resultados, classificou-se a amostra em quatro categorias: i) portos com eficiência baixa, com grau de eficiência entre 0 e 0,25 ; ii) portos com eficiência média- 
baixa, com grau de eficiência entre 0,251 e 0,50; iii) portos com eficiência média-alta, com grau de eficiência entre 0,501 e 0,75 ; iv) portos com eficiência alta, com grau de eficiência entre 0,751 e 1,00 .

Dos 49 portos da amostra, 15 portos apresentaram eficiência baixa, ou seja, apresentaram resultados entre 0 e 0,25 . O porto com o grau de eficiência mais baixo foi o porto de Zeebrugge, localizado na Bélgica, com modelo de gestão portuária PÚBLICO/privado. Entre esses 15 portos, encontra-se o porto de Santos, localizado no Brasil, o qual apresenta uma eficiência de 0,249 e modelo de gestão portuária PÚBLICO/privado (landlord), onde o setor público é responsável pela titularidade e regulação e o privado pela operação. Observou-se, ainda, que entre os 15 portos com o menor grau de eficiência, nenhum deles está localizado na China. Na Tabela 1 estão relacionados os portos que apresentaram grau de eficiência entre 0 e 0,25 .

Tabela 1 - Relação dos portos com eficiência baixa $(0$ a 0,25$)$

\begin{tabular}{lc}
\hline \multicolumn{1}{c}{ Porto } & Eficiência \\
\hline Zeebrugge, Bélgica & 0,117 \\
Kobe, Japão & 0,145 \\
Los Angeles, Estados Unidos & 0,151 \\
Vancouver, Canadá & 0,163 \\
Savannah, Estados Unidos & 0,173 \\
Ambarli, Turquia & 0,188 \\
Yokohama, Japão & 0,191 \\
Shahid Rajaee, Irã & 0,195 \\
Nagoya, Japão & 0,196 \\
Laem Chabang, Tailândia & 0,203 \\
Algeciras Bay, Espanha & 0,214 \\
New York- New Jersey, USA & 0,219 \\
Ho Chin Minh, Vietnam & 0,236 \\
Gioia Tauro, Itália & 0,247 \\
Santos, Brasil & 0,249 \\
\hline Média & $\mathbf{0 , 1 9 2 5}$ \\
\hline
\end{tabular}

Dos 49 portos da amostra, 19 apresentaram eficiência média-baixa, ou seja, eficiência entre 0,251 e 0,50. O primeiro porto da tabela, com grau de eficiência de 0,263, é o porto Felixstowe, localizado no Reino Unido. Dentre toda a amostra, este é o único porto que apresenta um modelo de gestão portuário totalmente privado, o que significa, conforme a classificação de Baird, que toda a gestão e administração portuária desse porto é controlada por empresas privadas.

Verificou-se também que somente um porto dos 19 com eficiência média-baixa está localizado na China. Na Tabela 2 estão relacionados os portos com eficiência média baixa, com grau de eficiência entre 0,251 e 0,50. 
Tabela 2 - Relação dos portos com eficiência média-baixa $(0,251$ a 0,50$)$

\begin{tabular}{lc}
\hline \multicolumn{1}{c}{ Porto } & Eficiência \\
\hline Felixstowe, Reino Unido & 0,263 \\
Valencia, Espanha & 0,265 \\
Jeddah, Arábia Saudita & 0,265 \\
Bremerhaven, Alemanha & 0,272 \\
Long Beach, EUA & 0,277 \\
Tokyo, Japão & 0,284 \\
Antwerp, Bélgica & 0,295 \\
Hamburg, Alemanha & 0,299 \\
Durban, África do Sul & 0,307 \\
Tanjung Perak & 0,328 \\
Colombo, Sri Lanka & 0,350 \\
Dalian, China & 0,355 \\
Jawaharlal Nehru, Índia & 0,357 \\
Port Said, Egito & 0,371 \\
Khor Fakkan/Sharjah, AE & 0,392 \\
Salalah, Omã & 0,400 \\
Tanjung Priok, Indonesia & 0,404 \\
Rotterdam, Holanda & 0,421 \\
Tanjung Pelepas, Malásia & 0,438 \\
\hline Média & $\mathbf{0 , 3 3 4}$ \\
\hline
\end{tabular}

Dos 49 portos da amostra, apenas nove apresentaram eficiência média-alta, ou seja, resultados entre 0,501 a 0,75. Dentre esses nove portos, cinco estão localizados na China. Dos cinco portos chineses com eficiência média-alta, os portos Kaohsiung, Xiamen e Yingkou possuem modelo de gestão PÚBLICO/ privado. Os outros dois portos (Guangzhou Harbor e Tianjin) apresentam modelo de gestão totalmente público, ou seja, além de o setor público regular e ter a posse da propriedade, o mesmo é responsável também pelos serviços de movimentação da carga. Na Tabela 3 estão relacionados os portos com eficiência média-alta - com grau de eficiência variando entre 0,501 e 0,75 .

Tabela 3 - Relação dos portos com eficiência média-alta $(0,501$ a 0,75$)$

\begin{tabular}{lc}
\hline \multicolumn{1}{c}{ Porto } & Eficiência \\
\hline Port Klang, Malásia & 0,511 \\
Dubai, Emirados Árabes & 0,524 \\
Busan, Coreia do Sul & 0,565 \\
Kaohsiung, Taiwan, China & 0,583 \\
Tianjin, China & 0,641 \\
Xiamen, China & 0,659 \\
Balboa, Panamá & 0,660 \\
Yingkou, China & 0,710 \\
Guangzhou Harbor, China & 0,735 \\
\hline Média & $\mathbf{0 , 6 2 1}$ \\
\hline
\end{tabular}


Dos 49 portos da amostra, apenas seis portos apresentaram eficiência alta, ou seja, resultados entre 0,751 e 1,00. Os portos de Shangai, Singapura, Hong Kong, Ningbo e Lianyungang apresentaram grau máximo de eficiência $(1,00)$.

Dentre os seis portos considerados eficientes, encontram-se três diferentes modelos de gestão, segundo a classificação utilizada por Baird (1995, 1997). O porto de Shangai e o porto de Lianyungang apresentam modelo de gestão PÚBLICO/privado. Em oposição a esse modelo, encontram-se os portos de Hong Kong e Ningbo, que apresentam modelo de gestão portuária PRIVADO/público. Já os portos de Singapura e Qingdao apresentam modelo de gestão portuário totalmente público. Destaca-se também que, dentre os seis portos com eficiência alta, apenas o porto de Singapura não está localizado na China. Na Tabela 4 estão relacionados os portos com eficiência alta, com grau de eficiência entre 0,751 e 1,00.

Tabela 4 - Relação dos portos com eficiência alta $(0,751$ a 1,00$)$

\begin{tabular}{lc}
\hline \multicolumn{1}{c}{ Porto } & Eficiência \\
\hline Shangai, China & 1,000 \\
Singapore, Singapura & 1,000 \\
Hong Kong, China & 1,000 \\
Ningbo- Zhoushan, China & 1,000 \\
Lianyungang, China & 1,000 \\
Qingdao, China & 0,781 \\
\hline Média & $\mathbf{0 , 9 6 3}$ \\
\hline
\end{tabular}

\subsection{Síntese dos portos referência (peers)}

Através do DEA, também é possível indicar para quais portos da amostra os portos com eficiência máxima são referência (peers). No Apêndice B estão relacionados os respectivos peers para cada porto da amostra. A Tabela 5 apresenta o resumo da contagem de peers, indicando a quantidade de portos para os quais cada porto com grau de eficiência máxima foi referência.

Tabela 5 - Resumo contagem de peers DEA

\begin{tabular}{ccc}
\hline DMUs & Portos & Peer Count Summary \\
\hline 1 & Shangai, China & 36 \\
2 & Singapore, Singapura & 06 \\
3 & Hong Kong, China & 19 \\
5 & Ningbo- Zhoushan, China & 35 \\
28 & Lianyungang, China & 24 \\
\hline
\end{tabular}

O porto de Shangai foi referência para o maior número de portos, 36. Em seguida, aparece o porto de Ningbo- Zhoushan, o qual foi referência para 35 portos. Os portos de Lianyungang e Hong 
Kong foram referência para 24 e 19 portos, respectivamente. Por último, aparece o porto de Singapura, o qual foi referência para seis portos.

\section{Conclusões}

O estudo realizado sobre a relação entre modelo de gestão e eficiência em portos de contêineres buscou analisar se existe uma relação entre essas duas variáveis. Através do método DEA, foram apontados os portos mais eficientes dentro da amostra. Com esses resultados, identificaram-se os modelos de gestão portuária desses portos para então verificar se havia uma relação entre eficiência e modelo de gestão.

Em seu estudo, Tongzon e Heng (2005) pesquisaram a relação entre privatização e eficiência portuária. Eles concluíram que a participação do setor privado na indústria portuária contribui para um aperfeiçoamento na eficiência operacional do porto. Porém, eles também afirmam que uma total privatização dos portos não é uma maneira eficaz de aumentá-la, o que significa que a relação entre eficiência e privatização não é linear.

Já Liu (1995) e Notteboom, Coeck e Van-Den Broech (2000) chegaram a uma conclusão contrária: de que o modelo de gestão não possui efeito significativo na eficiência portuária. Nessa mesma linha, Valentine e Gray (2002) também concluíram em seu estudo que a eficiência não está relacionada com a titularidade portuária.

Corroborando o que foi encontrado na literatura da área, conclui-se, a partir do presente estudo, que não há uma relação direta entre modelo portuário e eficiência, já que, entre os seis portos considerados eficientes, foram encontrados três diferentes modelos de gestão, segundo a classificação utilizada por Baird $(1995,1997)$. Além disso, o único porto com gestão totalmente privada na amostra considerada, o porto de Felixstowe (Reino Unido), apresentou eficiência médiabaixa, evidenciando que a privatização total do porto não é necessariamente sinônimo de maior eficiência.

\section{1 Implicações Gerenciais}

Frente à grande expansão da globalização e das trocas comerciais internacionais, muitos portos de contêineres veem a necessidade de revisarem frequentemente sua capacidade, no intuito de garantir um fornecimento de serviços satisfatórios aos seus clientes e, assim, manter sua competitividade. Muitas vezes, a necessidade de construir um novo terminal ou de aumentar a capacidade dos já existentes torna-se inevitável, porém, antes de os portos tomarem essa decisão, é de grande importância que analisem se estão usando adequadamente suas facilidades e se os 
produtos (outputs) estão sendo maximizados, dados os recursos (inputs) existentes (CULLINANE et al, 2005).

Este trabalho proporciona resultados que apontam grandes diferenças de eficiência entre os portos, com base em seus inputs e outputs, sugerindo que um melhor gerenciamento e planejamento portuário podem proporcionar um aumento significativo de sua eficiência, não sendo muitas vezes necessário o aumento de seus recursos, como por exemplo, a construção de um novo terminal.

Como mostrado nas conclusões do trabalho, o modelo de gestão privado não está necessariamente relacionado com uma maior eficiência portuária e, portanto, a privatização nem sempre é a solução para o aumento da eficiência. Vieira, Kliemann Neto e Monfort (2013) e Milan e Vieira (2011) apontam em seus estudos a importância de ações de governança para o aumento da eficiência portuária, eficiência esta que é de fundamental importância para o aumento da competitividade das diversas empresas importadoras e exportadoras de um país, o que acaba impactando na economia como um todo.

Por fim, os resultados também promovem reflexões acerca das diferenças de eficiência entre os portos do continente asiático e os localizados em outros continentes, uma vez que, todos os portos com eficiência máxima estão localizados naquele continente.

\subsection{Limitações do Estudo e Sugestões de Pesquisas Futuras}

Este estudo considerou dados de 49 portos entre o 50 com maior movimentação de contêineres em 2010, tendo sido coletados dados de dois inputs - comprimento do cais em metros e área total do terminal em $\mathrm{m}^{2}$ - e um output - movimentação anual em TEUs. Além disso, somente um modelo de DEA - o modelo DEA VRS orientado a output - foi utilizado. Observa-se que a utilização de outro modelo de DEA, a alteração da amostra ou adição de outros outputs e inputs poderiam alterar significativamente os resultados.

O porto de Shenzhen foi excluído da pesquisa, já que os dados dos seus inputs não foram encontrados na fonte consultada. Também não foram encontrados nas fontes de pesquisa os modelos de gestão, segundo a estrutura proposta por Baird $(1995,1997)$, de toda a amostra. Porém, foi possível encontrar os modelos de gestão portuária de todos os portos que apresentaram grau de eficiência alto e também do porto que apresentou o menor grau de eficiência da amostra. Outra limitação do estudo foi a soma da área total disponível em $\mathrm{m}^{2}$, já que, nos terminais que possuíam mais de um tipo de cais, além do cais para contêineres, não foi possível identificar a área específica em metros quadrados utilizada para a movimentação de contêineres. 
Para pesquisas futuras sugere-se a variação da amostra, a adição de mais inputs e outputs e a aplicação dos outros modelos de DEA. Com essa mesma amostra, mais três modelos de DEA podem ser aplicados e, com isso, os resultados podem ser comparados, ampliando ainda mais o conhecimento sobre a eficiência dos portos estudados. Além disso, as classificações dos portos quanto a sua posição geográfica na rede global de transporte marítimo e seus modelos de governança podem ser considerados, ampliando o estudo e as relações dessas variáveis com o grau de eficiência portuária.

\begin{abstract}
Nowadays, the harbors are part of a complex logistics chain with many combinations related to its ownership, management model and governance. Considering this context, this research has as its goal to analyze the relationship between the port management model and the effectiveness in containers port. Therefore, having as basis the data of the 50 greatest containers ports in the world, it was applied a DEA VRS model orientating the output to identify the harbors which are more effective and their respective management models were analyzed. Based on these results, the harbors were gathered as the effectiveness degree (high, medium-high, medium-low or low) and as the management model (totally public, predominantly public, predominantly private or totally private). The results indicate that there is no direct difference between the port management model and effectiveness, considering that among the six harbors with high effectiveness degree were identified different management models.
\end{abstract}

Key-words: Ports; Management; Models; Efficiency; Data Envelopment Analysis.

\title{
Referências
}

BAIRD, A. UK port privatization. In: UK PORT PRIVATISATION CONFERENCE, 2005, Edinburgh. Proceedings of UK Port Privatization Conference, Scottish Transport Studies Group, Edinburgh, 1995.

BAIRD, A. Port privatization: An analytical framework. In: INTERNATIONAL ASSOCIATION OF MARITIME ECONOMISTS - IAME, 1997. IAME 1997 Conference Proceedings, Londres, 1997.

BARROS, C. P.; ATHANASSIOU, M. Efficiency in European seaports with DEA: evidence from Greece and Portugal. Maritime Economics and Logistics, v. 6, n. 1, p. 122-140, 2004. cross ref

BARROS, C. P. Decomposing Growth in Portuguese Seaports: A Frontier Cost Approach. Maritime Economics and Logistics, v. 7, n. 1, p. 297-315, 2005. crossref

BARROS, C. P. Productivity Assessment of African Seaports. African Development Review, v. 24, n. 1, p. 67-78, 2012. cross ref

BICHOU, K; GRAY, R. A critical review of conventional terminology for classifying seaports. Transportation Research Part A, v. 39, n. 1, p.75-92, 2005. crossref

BICHOU, K. An empirical study of the impacts of operating and market conditions on container- port efficiency and benchmarking. Research in Transportation Economics, v. 42, n. 1, p. 28-37, 2013. crossref

CHARNES, A.; COOPER, W.W; RHODES, E. Measuring the efficiency of decision making units. European Journal of Operational Research, v. 2, p. 429-444, 1978. crossref

COELLI, T. A Guide to DEAP Version 2.1: a Data Envelopment Analysis (Computer) Program. Centre for Efficiency and Productivity Analysis, Department of Econometrics, University of New England, New England, 1996.

COLLIS, J.; HUSSEY, R. Pesquisa em administração: um guia para alunos de graduação e pós-graduação. 2. ed. Porto Alegre: Bookman, 2005. 
CULLINANE, K.; SONG, D. W.; GRAY, R. A stochastic frontier model of the efficiency of major container terminals in Asia: assessing the influence of administrative and ownership structures. Transportation Research Part A, v. 36, n. 1, p.743-762, 2002. crossref

CULLINANE, K.; JI, P.; WANG, T. F. The relationship between privatization and DEA estimates of efficiency in the container port industry. Journal of Economics and Business, v. 57, n. 1, p. 433-462, 2005. crossref

CULLINANE, K.; SONG, D. W.; WANG, T. The application of mathematical programming approaches to estimating container port production efficiency. Journal of Productivity Analysis, v. 24, n. 1, p. 73-92, 2005. crossref

CULLINANE, K.; SONG, D. W. Estimating the Relative Efficiency of European Container Ports: A Stochastic Frontier Analysis. Research in Transportation Economics, v. 16, n. 1, p. 85-115, 2006. crossref

CULLINANE, K.; WANG, T. F. Data Envelopment analysis (DEA) and improving container port efficiency. Research in Transportation Economics, v. 17, n. 1, p. 517-566, 2007.

GONZALEZ, M. M.; TRUJILLO, L. Reforms and Infrastructure Efficiency in Spain's Container Ports. Transportation Research Part A, v. 42, n. 1, p. 243-257, 2008. crossref

HUNG, S. W.; LU, W. M.; WANG, T. P. Benchmarking the operating efficiency of Asia container ports. European Journal of Operational Research, v. 203, n. 1, p. 706-713, 2010. crossref

de KOSTER, M. B. M; BALK, B. M.; van NUS, W. T. I. On Using DEA for Benchmarking Container Terminals. International Journal of Operating \& Production Management, v. 29, n. 11, p. 1140-1155, 2009. crossref

LAKATOS, E. M.; MARCONI, M. A. Metodologia do trabalho científico: procedimentos básicos, pesquisa bibliográfica, projeto e relatório, publicações e trabalhos científicos. 7. ed. São Paulo: Atlas, 2007.

LIN, L. C; TSENG, L. A. Application of DEA and SFA on the measurement of operating efficiencies for 27 international container ports. Proceedings of the Eastern Asia Society for Transportation Studies, v. 5, p. 592-607, 2005.

LIU, Z. Ownership and productive efficiency: With reference to British ports. Tese (PhD) - Queen Mary and Westfield College University of London, Londres, 1992.

LIU, Z. The Comparative Performance of Public and Private Enterprises: The case of British Ports. Journal of Transport Economics and Policy, v. 3, n. 29, p. 263-274, 1995.

LIU, Q. Efficiency Analysis of Container Ports and Terminals. Tese (PhD), Department of Civil, Environmental And Geomatic Engineering, University College London, Londres, 2010.

LOZANO, S.; VILLA, G.; CANCA, D. Application of centralised DEA approach to capital budgeting in Spanish ports. Computers \& Industrial Engineering, Spain, v. 60, n. 3, p. 455-465, 2011. crossref

MERK, O.; DANG, T. Efficiency of world ports in container and bulk cargo (oil, coal, ores and grain). OECD Regional Development Working Papers, 2012/09. OECD Publishing. Disponível em: http://dx.doi.org/10.1787/sk92vgw39zs2en. Acesso em: 17.jun.2014.

MILAN, G. S.; VIEIRA G. B. B. Proposição de um modelo conceitual em torno da prática da governança em cadeias logístico-portuárias. Revista Gestão Industrial, v. 7, n. 4, p. 154-174, 2011.

NIAVIS, S.; TSEKERIS, T. Ranking and causes of inefficiency of container seaports in South-Eastern Europe. European Transport Research Review, v. 4, n. 4, p. 235-244, 2012. crossref

NOTTEBOOM, T.; COECK, C.; VAN DEN BROECH, J. Measuring and Explaining the relative Efficiency of Container Terminals by Means of Bayesian Stochastic Frontier Models. International Journal of Maritime Economics, v. 2, n. 2, p.83-106, 2000.

SHARMA, M. J.; YU, S. J. Performance based stratification and clustering for benchmarking of container terminals. Expert Systems with Applications, v. 36, n. 3, p. 5016-5022, 2009. crossref

SHARMA, M. J.; YU, S. J. Benchmark optimization and attribute identification for improvement. European Journal of Operational Research, n. 201, n. 2, p. 568-580, 2010. crossref 
SOUSA JÚNIOR, J. N. C. Avaliação da eficiência dos portos utilizando análise envoltória de dados: Estudo de caso dos portos da região nordeste do Brasil. Dissertação (Mestrado). Curso de Engenharia de Transportes, Departamento de Centro de Tecnologia, Universidade Federal do Ceará, Fortaleza, Ceará, 2010.

SUN, X.; YAN, Y.; LIU, J. Econometric Analysis of Technical Efficiency of Global Container Operators. $11^{\text {th }}$ INTERNATIONAL CONFERENCE OF HONG KONG SOCIETY FOR TRANSPORTATION STUDIES. Proceedings of the $1^{\text {th }}$ International Conference of Hong Kong Society for Transportation Studies: Sustainable Transportation, p. 667-676, 2006.

TONGZON, J.; HENG, W. Port Privatization, Efficiency and Competitiveness: Some Empirical Evidence from Container Ports (Terminals). Transportation Research Part A, v. 39, n. 5, p. 405-424, 2005. crossref

TRUJILlO, L.; TOVAR, B. The European Port Industry: An Analysis of its Economic Efficiency. Maritime Economics and Logistics, v. 9, n. 2, p. 148-171, 2007. cross ref

TRUJILLO, L.; GONZÁLEZ, M. M.; JIMÉNEZ, J. L. An overview on the reform process of African ports. Utilities Policy, v. 25, n. 1, p.12-22, 2013. crossref

VALENTINE, V. F.; GRAY, R. An Organisational Approach to Port Efficiency. In: INTERNATIONAL ASSOCIATION OF MARITIME ECONOMISTS - IAME. IAME 2002 Conference Proceedings, Panamá, 2002. VIEIRA, G. B. B. Modelo de governança aplicado a cadeias logístico-portuárias. Tese (Doutorado). Programa de Pós Graduação em engenharia de Produção. Porto Alegre, 2013.

VIEIRA, G. B. B; KLIEMANN NETO, F. J; MONFORT, A. Gobernanza en cadenas logístico-portuarias de contenedores: proposición de un modelo conceptual. Revista Espacios, v. 34, n. 5, p. 8, 2013.

WANG, T. F.; SONG, D. W.; CULLINANE, K. The Applicability of Data Envelopment Analysis to Efficiency Measurement of Container Port. PROCEEDINGS. In: INTERNATIONAL ASSOCIATION OF MARITIME ECONOMISTS - IAME. IAME 2002 Conference Proceedings, Panamá, 2002.

WANG, T. F.; CULLINANE, K. The efficiency of European container terminals and implications for supply chain management. Maritime Economics and Logistics, v. 8, n. 1, p. 82-99, 2006. crossref

WANKE, P. F. Physical infrastructure and shipment consolidation efficiency drivers in Brazilian ports: A two-stage network- DEA approach. Transport Policy, v. 29, n. 1, p.145-153, 2013.

WORLD BANK. Public-Private Infrastructure Advisory Facility. Port Reform Toolkit. Second Edition. Disponível em: http://www.ppiaf.org/documents/toolkits/Portoolkit/Toolkit/index.html. Acesso em: 14.mai.2013.

WU, Y. C. J.; GOH, M. Container port efficiency in emerging and more advanced markets. Transportation Research Part E, v. 46, n. 6, p. 1030-1042, 2010. crossref

YUEN, A. C. L.; ZHANG, A.; CHEUNG, W. Foreign participation and competition: A way to improve the container port efficiency in China? Transportation Research Part A, v. 49, n. 1, p. 220-231, 2013. 
APÊNDICE A - Dados coletados da pesquisa

\begin{tabular}{|c|c|c|c|c|}
\hline \multirow[b]{2}{*}{ DMUs } & \multirow[b]{2}{*}{ Portos } & \multicolumn{2}{|c|}{ INPUTS } & \multirow{2}{*}{$\begin{array}{c}\text { OUTPUTS } \\
\text { Milhões de TEUs } \\
(2010)\end{array}$} \\
\hline & & $\begin{array}{c}\text { Área do } \\
\text { terminal }\left(\mathbf{m}^{2}\right)\end{array}$ & $\begin{array}{l}\text { Comprimento } \\
\text { do cais }(\mathbf{m})\end{array}$ & \\
\hline 1 & Shangai, China & $8.569 .837,00$ & $8.956,00$ & 29.069 .000 \\
\hline 2 & Singapura & $6.233 .000,00$ & $17.410,00$ & 28.431 .100 \\
\hline 3 & Hong Kong, China & $3.438 .500,00$ & $8.409,00$ & 23.699 .242 \\
\hline 4 & Shenzhen, China & $\mathrm{N} / \mathrm{D}$ & N/D & 22.509 .700 \\
\hline 5 & Busan, Coreia do Sul & $4.277 .778,00$ & $14.610,00$ & 14.194 .334 \\
\hline 6 & Ningbo- Zhoushan, China & 757.000 & $3.748,00$ & 13.144 .000 \\
\hline 7 & Guangzhou Harbor, China & $4.604 .600,00$ & $4.950,00$ & 12.550 .000 \\
\hline 8 & Qingdao, China & $1.322 .800,00$ & $5.449,00$ & 12.012 .000 \\
\hline 9 & Dubai, Emirados Árabes & $3.536 .905,00$ & $7.475,00$ & 11.600 .000 \\
\hline 10 & Rotterdam, Holanda & $5.662 .000,00$ & $10.900,00$ & 11.145 .804 \\
\hline 11 & Tianjin, China & $1.859 .400,00$ & $4.674,00$ & 10.080 .000 \\
\hline 12 & Kaohsiung, Taiwan, China & $1.416 .374,00$ & $6.713,00$ & 9.181 .211 \\
\hline 13 & Port Klang, Malásia & $1.824 .300,00$ & $7.570,00$ & 8.870 .000 \\
\hline 14 & Antwerp, Bélgica & $7.384 .073,00$ & $15.412,00$ & 8.468 .475 \\
\hline 15 & Hamburg, Alemanha & $5.923 .550,00$ & $9.148,00$ & 7.900 .000 \\
\hline 16 & Tanjung Pelepas, Malásia & $1.800 .000,00$ & $4.320,00$ & 6.530 .000 \\
\hline 17 & Long Beach, Estados Unidos & $4.452 .227,00$ & $7.323,00$ & 6.263 .399 \\
\hline 18 & Xiamen, China & $480.000,00$ & $2.483,00$ & 5.820 .000 \\
\hline 19 & New York-New Jersey, Estados Unidos & $5.566 .100,00$ & $7.615,00$ & 5.292 .020 \\
\hline 20 & Dalian, China & $2.048 .579,00$ & $4.253,00$ & 5.242 .000 \\
\hline 21 & Laem Chabang, Tailândia & $4.313 .800,00$ & $10.300,00$ & 5.068 .076 \\
\hline 22 & Bremerhaven, Alemanha & $4.359 .000,00$ & $5.259,00$ & 4.871 .297 \\
\hline 23 & Jawaharlal Nehru, Índia & $2.140 .000,00$ & $3.749,00$ & 4.752 .000 \\
\hline 24 & Tanjung Priok, Indonésia & $1.666 .000,00$ & $3.192,00$ & 4.714 .857 \\
\hline 25 & Tokyo, Japão & $1.521 .547,00$ & $4.479,00$ & 4.284 .944 \\
\hline 26 & Valência, Espanha & $1.814 .900,00$ & $4.793,00$ & 4.206 .937 \\
\hline 27 & Los Angeles, Estados Unidos & $6.477 .368,00$ & $9.381,00$ & 4.078 .198 \\
\hline 28 & Colombo, Sri Lanka & $720.300,00$ & $3.154,00$ & 4.000 .000 \\
\hline 29 & Lianyungang, China & $162.000,00$ & 540,00 & 3.870 .000 \\
\hline 30 & Ho Chin Minh, Vietnam & $2.389 .000,00$ & $4.788,00$ & 3.856 .000 \\
\hline 31 & Jeddah, Arábia Saudita & $3.136 .071,00$ & $4.100,00$ & 3.830 .857 \\
\hline 32 & Salalah, Omã & $765.000,00$ & $2.205,00$ & 3.485 .395 \\
\hline 33 & Port Said, Egito & $1.442 .130,00$ & $2.400,00$ & 3.474 .792 \\
\hline 34 & Felixstowe, Reino Unido & $1.522 .000,00$ & $3.638,00$ & 3.400 .000 \\
\hline 35 & Yingkou, China & $240.000,00$ & 826,00 & 3.338 .000 \\
\hline 36 & Yokohama, Japão & $2.094 .922,00$ & $5.390,00$ & 3.280 .191 \\
\hline 37 & Tanjung Perak & $1.100 .000,00$ & $2.370,00$ & 3.030 .000 \\
\hline 38 & Khor Fakkan/Sharjah, Emirados Árabes & $525.000,00$ & $1.860,00$ & 3.022 .524 \\
\hline 39 & Gioia Tauro, Itália & $1.600 .000,00$ & $3.145,00$ & 2.851 .261 \\
\hline 40 & Savannah, Estados Unidos & $5.666 .000,00$ & $4.713,00$ & 2.825 .179 \\
\hline 41 & Algeciras, Espanha & $1.147 .459,00$ & $3.726,00$ & 2.810 .242 \\
\hline 42 & Balboa, Panamá & $182.000,00$ & $1.511,00$ & 2.758 .506 \\
\hline 43 & Santos, Brasil & $833.000,00$ & $2.980,00$ & 2.722 .225 \\
\hline 44 & Shahid Rajaee, Irã & $1.450 .000,00$ & $3.777,00$ & 2.592 .522 \\
\hline 45 & Kobe, Japão & $1.889 .497,00$ & $7.275,00$ & 2.556 .291 \\
\hline 46 & Nagoya, Japão & $1.405 .549,00$ & $3.670,00$ & 2.548 .851 \\
\hline 47 & Ambarli, Turquia & $848.606,00$ & $5.690,00$ & 2.540 .353 \\
\hline 48 & Durban, África do Sul & $1.840 .000,00$ & $2.000,00$ & 2.529 .209 \\
\hline 49 & Vancouver, Canadá & $1.783 .312,00$ & $4.560,00$ & 2.514 .309 \\
\hline 50 & Zeebrugge, Bélgica & $2.922 .000,00$ & $6.860,00$ & 2.389 .879 \\
\hline
\end{tabular}

Fonte: Containerisation International 2012 
APÊNDICE B - Relação dos peers

\begin{tabular}{|c|c|c|}
\hline DMUs & Portos & Peers \\
\hline 1 & Shangai, China & 1 \\
\hline 2 & Singapore, Singapura & 2 \\
\hline 3 & Hong Kong, China & 3 \\
\hline 4 & Busan, Coreia do Sul & 23 \\
\hline 5 & Ningbo- Zhoushan, China & 5 \\
\hline 6 & Guangzhou Harbor, China & 281 \\
\hline 7 & Qingdao, China & 35 \\
\hline 8 & Dubai, Emirados Árabes & 135 \\
\hline 9 & Rotterdam, Holanda & $\begin{array}{lll}3 & 1 & 2 \\
\end{array}$ \\
\hline 10 & Tianjin, China & $\begin{array}{lll}3 & 1 & 5 \\
\end{array}$ \\
\hline 11 & Kaohsiung, Taiwan, China & 35 \\
\hline 12 & Port Klang, Malásia & 35 \\
\hline 13 & Antwerp, Bélgica & 12 \\
\hline 14 & Hamburg, Alemanha & 123 \\
\hline 15 & Tanjung Pelepas, Malásia & $28 \quad 1 \quad 5$ \\
\hline 16 & Long Beach, Estados Unidos & 135 \\
\hline 17 & Xiamen, China & 528 \\
\hline 18 & New York- New Jersey, Estados Unidos & $\begin{array}{lll}3 & 1 & 5 \\
\end{array}$ \\
\hline 19 & Dalian, China & $28 \quad 15$ \\
\hline 20 & Laem Chabang, Tailândia & $\begin{array}{lll}3 & 1 & 2 \\
\end{array}$ \\
\hline 21 & Bremerhaven, Alemanha & $28 \quad 1 \quad 5$ \\
\hline 22 & Jawaharlal Nehru, India & $28 \quad 15$ \\
\hline 23 & Tanjung Priok, Indonesia & 1528 \\
\hline 24 & Tokyo, Japão & $\begin{array}{lll}3 & 5 & 1 \\
\end{array}$ \\
\hline 25 & Valencia, Espanha & $\begin{array}{lll}3 & 1 & 5 \\
\end{array}$ \\
\hline 26 & Los Angeles, Estados Unidos & 123 \\
\hline 27 & Colombo, Sri Lanka & 1528 \\
\hline 28 & Lianyungang, China & 28 \\
\hline 29 & Ho Chin Minh, Vietnam & 1528 \\
\hline 30 & Jeddah, Arábia Saudita & 1528 \\
\hline 31 & Salalah, Omã & $28 \quad 15$ \\
\hline 32 & Port Said, Egito & $28 \quad 1 \quad 5$ \\
\hline 33 & Felixstowe, Reino Unido & 1528 \\
\hline 34 & Yingkou, China & $\begin{array}{lll}1 & 5 & 28 \\
\end{array}$ \\
\hline 35 & Yokohama, Japão & $\begin{array}{lll}3 & 1 & 5 \\
\end{array}$ \\
\hline 36 & Tanjung Perak & $28 \quad 1 \quad 5$ \\
\hline 37 & Khor Fakkan/Sharjah, Emirados Árabes & $\begin{array}{lll}1 & 5 & 28 \\
\end{array}$ \\
\hline 38 & Gioia Tauro, Itália & $28 \quad 1 \quad 5$ \\
\hline 39 & Savannah, Estados Unidos & 281 \\
\hline 40 & Algeciras Bay, Espanha & 1528 \\
\hline 41 & Balboa, Panamá & 528 \\
\hline 42 & Santos, Brasil & $\begin{array}{lll}1 & 5 & 28 \\
\end{array}$ \\
\hline 43 & Shahid Rajaee, Irã & $28 \quad 1 \quad 5$ \\
\hline 44 & Kobe, Japão & 35 \\
\hline 45 & Nagoya, Japão & $\begin{array}{lll}1 & 5 & 28 \\
\end{array}$ \\
\hline 46 & Ambarli, Turquia & 35 \\
\hline 47 & Durban, África do Sul & 128 \\
\hline 48 & Vancouver, Canadá & 135 \\
\hline 49 & Zeebrugge, Bélgica & 135 \\
\hline
\end{tabular}

Fonte: Resultados da pesquisa 


\section{Dados dos autores}

Nome completo: Priscila Milani

Filiação institucional: Universidade de Caxias do Sul (UCS)

Departamento: Centro de Ciências Sociais (CCSO)

Função ou cargo ocupado: Ex-aluna do Curso de Comércio Internacional

Endereço completo para correspondência: Rua Francisco Getúlio Vargas, 1130 - CEP 95070-560 -

Caxias do Sul

Fone: (+5554) 3218-2100

e-mail: prikamil@hotmail.com

Nome completo: Guilherme Bergmann Borges Vieira

Filiação institucional: Universidade de Caxias do Sul (UCS)

Departamento: Centro de Ciências Sociais (CCSO)

Função ou cargo ocupado: Professor do Curso de Comércio Internacional

Endereço completo para correspondência: Rua Francisco Getúlio Vargas, 1130 - CEP 95070-560 -

Caxias do Sul

Fone: +55 $543218-2100$

e-mail: gbbvieir@ucs.br

Nome completo: Arturo Monfort Mulinas

Filiação institucional: Fundación Valenciaport (FV)

Departamento: Departamento de Pesquisa, Desenvolvimento e Inovação

Função ou cargo ocupado: Diretor de Pesquisa, Desenvolvimento e Inovação

Endereço completo para correspondência: Sede APV - Fase III, Avda. del Muelle del Turia, s/n 46024 - Valencia - España

Telefones para contato: +34963939400

e-mail: amonfort@fundacion.valenciaport.com

Nome completo: Fábio Verruck

Filiação institucional: Universidade de Caxias do Sul (UCS)

Departamento: Centro de Ciências Sociais (CCSO)

Função ou cargo ocupado: Coordenador do Curso de Comércio Internacional

Endereço completo para correspondência: Rua Francisco Getúlio Vargas, 1130 - CEP 95070-560 -

Caxias do Sul

Fone: +55 54 3218-2100 
e-mail:fverruck@ucs.br

Nome completo: Roberto Birch Gonçalves

Filiação institucional: Universidade de Caxias do Sul (UCS)

Departamento: Centro de Ciências Sociais (CCSO)

Função ou cargo ocupado: Professor do Curso de Comércio Internacional e Diretor do Instituto de Pesquisas Econômicas e Sociais (IPES)

Endereço completo para correspondência: Rua Francisco Getúlio Vargas, 1130 - CEP 95070-560 Caxias do Sul

Fone: +55 54 3218-2100

e-mail: rbgoncal@ucs.br

Submetido: 2014-06-26

Aceito em: 2014-12-18 